\title{
Análisis de la casuística de 5 años en la Unidad de Quemados del Hospital Luis Vernaza, Guayaquil, Ecuador
}

\section{Analysis of a 5-year casuistry in the Burn Unit of Luis Vernaza Hospital, Guavaquil, Ecuador}

\author{
Guerrero-Torbay, R.*, Palacios-Martínez, J. ${ }^{\star *}$, Salamea-Molina, P.***,

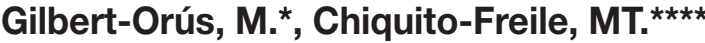

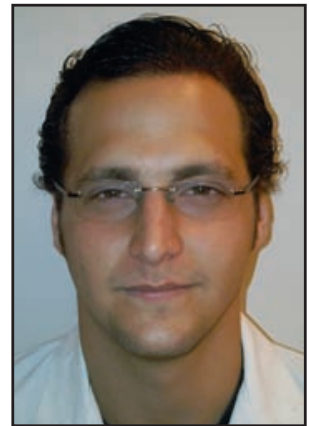

Guerrero-Torbay, R.

\section{Resumen}

En el periodo comprendido entre 2006 y 2010 registramos 646 ingresos a la Unidad de Quemados del Hospital Luis Vernaza, en Guayaquil, Ecuador. El género masculino fue más afectado que el femenino $(70,7 \%$ frente a 29,3\%). El domicilio fue el lugar donde más ocurrieron los accidentes. La llama fué la etiología más común $(\mathrm{n}=233)$. El 85\% de los pacientes ingresados requirió algún tipo de tratamiento quirúrgico. La estancia media hospitalaria no tuvo diferencias al comparar género o edad, pero fue mayor para aquellos pacientes con lesiones de más del $30 \%$ de la superficie corporal total $(46,9$ frente a 25,1 días; $p<0,05$ ) y en pacientes con quemaduras de tercer grado $(34,6$ frente a 20,8 días; $p<0,05)$.

Según los criterios de la American Burn Association, los pacientes con quemaduras de grado moderado a mayor tuvieron una estancia superior a la de los pacientes en la primera categoría $(p<0,05)$. No observamos diferencias entre los pacientes con quemaduras de grado moderado y mayor.

\begin{tabular}{|l}
\hline Palabras clave \begin{tabular}{l|} 
Quemaduras, Etiología, \\
Tratamiento, Casuística en quemados.
\end{tabular} \\
Nivel de evidencia científica IV
\end{tabular}

\section{Key words Burns, Etiology, \\ Treatment, Casuistry in burn patients.}

Level of evidence IV

* Médico Residente de Cirugía General, Hospital "Luis Vernaza”, Guayaquil, Ecuador.

** Jefe del Servicio de Cirugía Plástica, Reconstructiva, Estética y Quemados, Hospital "Luis Vernaza”, Guayaquil, Ecuador.

*** Especialista en Cirugía Plástica, Reconstructiva, Estética y Quemados, Hospital "Luis Vernaza", Guayaquil, Ecuador.

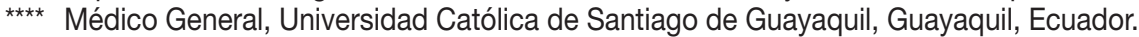




\section{Introducción}

Las quemaduras son lesiones secundarias a la acción de diversos agentes que esquemáticamente podemos clasificar en: físicos (térmicos, electricidad y radiación); químicos y biológicos. Todos ellos pueden provocar desde alteraciones funcionales reversibles, hasta la destrucción tisular total e irreversible. Por ser la piel nuestra superficie de contacto con el medio externo, representa el principal órgano afectado (1). Las quemaduras son el trauma más serio y devastador que le puede suceder a un ser humano (2). Incapacitan aproximadamente a unos 9 millones de personas en el mundo (3). Solo en los Estados Unidos se estima que, durante un año, el $1 \%$ de la población sufre una quemadura que requiere tratamiento por un profesional de la salud (4); además, entre 1,25 y 2 millones de personas son tratadas anualmente por algún tipo de quemadura y aproximadamente 50.000 pacientes requieren hospitalización (3), con una estancia aproximada de 1 día por cada $1 \%$ de superficie corporal quemada y con una mortalidad del $4 \%$, ya sea ésta producida por la quemadura en sí o por sus complicaciones (3).

En nuestro país, Ecuador, no se conoce la incidencia anual de pacientes que sufren este tipo de lesión, debido a que muchas quemaduras menores son tratadas de manera ambulatoria en clínicas u hospitales y no se recogen estadísticamente. La mitad de las personas afectadas solo requieren medidas de primeros auxilios y analgésicos, mientras que el resto necesita algún tratamiento ambulatorio u hospitalario. Muchas veces el pronóstico del paciente con quemadura depende del enfoque inicial que reciba en la primera asistencia sanitaria, por lo que es fundamental para el médico familiarizarse con su fisiopatología y manejo. Consideramos también muy importante identificar las principales causas por las que se producen las quemaduras y los factores relacionados con su morbilidad intrahospitalaria.

El objetivo del presente estudio es describir las características de los pacientes afectados por quemaduras y tratados en la Unidad de Quemados del Hospital Luis Vernaza de Guayaquil, Ecuador, e identificar las causas más frecuentes, los lugares dónde más ocurrieron estos accidentes y encontrar los posibles factores que se asocian a una mayor estancia hospitalaria de los pacientes.

\section{Material y método}

Realizamos un estudio de carácter retrospectivo y descriptivo, en el cual incluimos a todos los pacientes que ingresaron a través del área de Emergencia del Hospital Luis Vernaza de Guayaquil (Ecuador) y fueron admitidos en la Unidad de Quemados de la misma institución entre enero del 2006 y diciembre del 2010.

Los criterios de inclusión que tomamos en cuenta para este estudio son los siguientes: pacientes varones y mujeres que acudieron al Hospital Luis Vernaza ya sea como ingreso directo o transferidos desde otras unidades de salud y que fueron hospitalizados por quemaduras de cualquier tipo en la Unidad de Quemados del mencionado hospital, con una edad mayor de 14 años. Excluimos del estudio a aquellos pacientes menores de 14 años y a los que sufrieron quemaduras que no cumplían criterios de hospitalización.

Con la autorización previa del Jefe de Servicio de la Unidad de Quemados del hospital, procedimos a la revisión manual de los datos registrados en el libro de ingresos de dicha Unidad y llevamos a cabo la recolección de datos en un formulario diseñado para tal fin. Posteriormente, tabulamos los datos utilizando el programa informático Software Microsoft Excel 2007.

Las variables consideradas para el estudio fueron: género de los pacientes (masculino o femenino); edad (en años); días de hospitalización hasta el alta definitiva del paciente o hasta su fallecimiento; extensión y profundidad de las quemaduras; localización corporal de las lesiones; etiología del accidente; tratamiento recibido (médico o médico-quirúrgico); tipo de cirugía realizada (limpieza quirúrgica, injerto de piel, colgajo y/o amputación); lugar donde ocurrió el accidente y supervivencia.

Introdujimos todas estas variables en una hoja de cálculo de Excel y procedimos a la tabulación de los datos.

Clasificamos a los pacientes en una de las tres categorías propuestas por American Burn Association (ABA) (8), que contempla los siguientes grupos:

- Quemadura menor:

- $<15 \%$ de superficie corporal de $1^{\circ}$ o $2^{\circ}$ grado.

- $<2 \%$ de superficie corporal de $3^{\text {er }}$ grado.

- No afectación ocular, auricular, facial o genital.

- Se excluyen quemaduras de etiología eléctrica $(8,9)$.

- Quemadura moderada:

- $15-25 \%$ de superficie corporal de $1^{\circ}$ o $2^{\circ}$ grado.

- 2-10\% de superficie corporal de $3^{\text {er }}$ grado.

- No afectación ocular, auricular, facial o genital.

- Se excluyen quemaduras de etiología eléctrica $(8,9)$.

- Quemadura mayor:

- $>25 \%$ de superficie corporal de $1^{\circ}$ o $2^{\circ}$ grado.

- $>10 \%$ de superficie corporal de $3^{\text {er }}$ grado.

- Todas las quemaduras con afectación ocular, auricular, facial o genital.

- Todas las quemaduras de causa eléctrica $(8,9)$.

Empleamos el software SPSS para determinar la incidencia de las quemaduras y cuantificar el número de accidentes de acuerdo al género, etiología y lugar del accidente. Realizamos los cálculos para determinar la edad promedio y el rango de distribución, y llevamos a cabo correlación de los días de hospitalización, la edad y la superficie corporal afectada. Empleamos la prueba T de Student para comparar la estancia media hospitalaria entre los diferentes grupos analizados. 


\section{RESULTADOS}

\section{Características del grupo de estudio}

Durante los 5 años tomados en cuenta para el presente estudio contabilizamos un total de 646 pacientes, de los cuales $457(70,7 \%)$ eran varones y $189(29,3 \%)$ mujeres. En cuanto a las edades, la edad máxima fue de 53 años y la mínima de 21 años, teniendo como promedio una edad de 36,4 años.

\section{Etiología de las lesiones}

Los tipos más frecuentes de quemaduras de acuerdo al agente etiológico, fueron las quemaduras por llama $(n=233)$ y las de tipo eléctrico $(n=209)$, que en conjunto representaron el $68,4 \%$ de todas las quemaduras. Las quemaduras térmicas representaron el 24,3\% $(n=157)$ de las lesiones. El porcentaje restante se debió a agentes químicos $(n=30)$ o a fricción $(n=17)$ (Gráfico 1).

\section{Lugar de los accidentes}

El lugar más frecuente donde ocurrieron los accidentes fue el domicilio $(n=304)$ y en segundo lugar el trabajo $(n=287)$. En conjunto, representaron más del $90 \%$ de los casos. El porcentaje restante se debió a accidentes ocurridos en la vía pública (n=55) (Gráfico 2).

\section{Distribución corporal de las lesiones}

De los 646 pacientes, 412 presentaron lesiones en extremidades superiores; esta región constituyó la zona corporal más frecuentemente afectada. Las extremidades inferiores estuvieron afectadas en más del $50 \%$ de los casos; el torso en 267 casos y la cabeza y el cuello en casi un tercio de los pacientes. La zona corporal menos afectada por este tipo de lesiones fue la genital, registrándose sólo 14 casos con compromiso de dicha área (Gráfico 3).

Evidentemente, algunos pacientes presentaron afectación simultánea de más de una región corporal: el 65\% de los casos tuvo afectación de al menos 2 regiones corporales $(n=270)$; también hubo pacientes con $3(n=130)$ e incluso $4(n=22)$ regiones afectadas (Gráfico 4$)$.

\section{Tratamiento recibido}

El 85\% de los casos requirió algún tipo de intervención quirúrgica además del tratamiento médico estandarizado que se brinda en la Unidad. De estas intervenciones, la limpieza quirúrgica fue la más realizada (519 pacientes). El tratamiento quirúrgico también contempló la realización de injertos de piel en las zonas corporales más afectadas $(n=479)$, así como rotación de colgajos en 28 casos. Los pacientes que recibieron injerto de piel, fueron sometidos a limpiezas previas (promedio: $10 \mathrm{lim}$ piezas/paciente); la amputación fue necesaria en tan solo 20 de los 646 casos (3\%) (Gráfico 5).

\section{Estancia hospitalaria según criterios de la American Burn Association (ABA)}

Según los criterios ABA, 124 de los pacientes tuvieron quemaduras de grado menor, 111 de grado moderado y en 411 fueron consideradas de grado mayor. La estancia hospitalaria fue significativamente inferior (media $19,3 \pm 10,4$ días; $p<0,05)$ en los pacientes con quemadu-
Gráfico 1. Etiología de las lesiones. Unidad de Quemados del Hospital Luis Vernaza (Guayaquil, Ecuador). Período 2006 - 2010

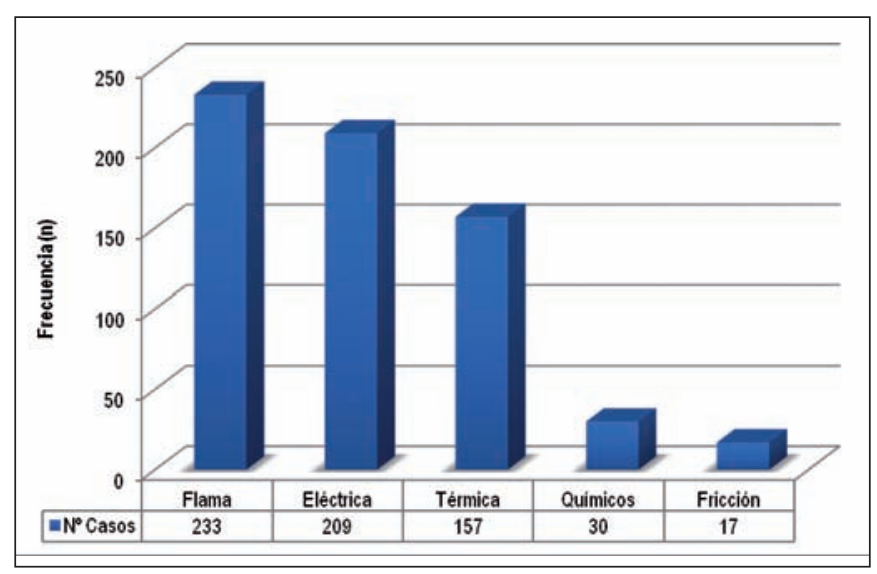

Gráfico 2. Lugar donde ocurrieron los accidentes. Unidad de Quemados del Hospital Luis Vernaza (Guayaquil, Ecuador). Período 2006 - 2010

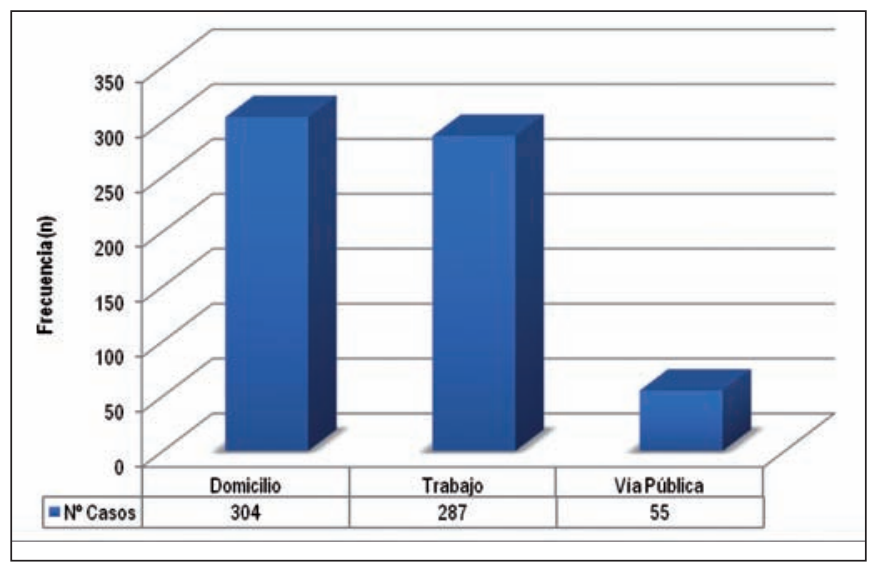

Gráfico 3. Distribución corporal de las lesiones. Unidad de Quemados del Hospital Luis Vernaza (Guayaquil, Ecuador) Período 2006 - 2010

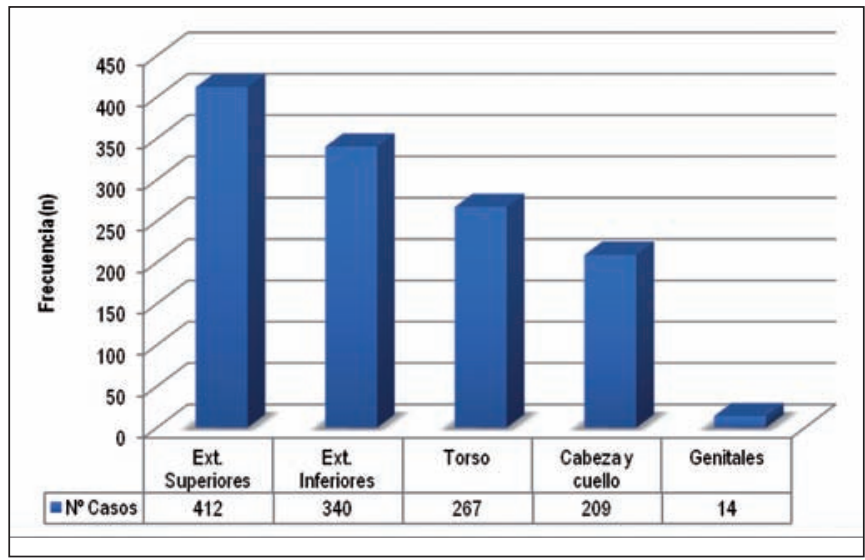

ras de grado menor en comparación con la de los pacientes con quemaduras de grado moderado $(28,9 \pm 14,47$ días) y grado mayor $(28,3 \pm 23,7)$. No registramos diferencias estadísticamente significativas en cuanto a la media de estancia hospitalaria entre los pacientes con quemaduras de grado moderado y mayor. La edad fue similar en los 3 grupos (Tabla I).

Más del $80 \%$ de los pacientes con quemaduras de grado menor tuvo una estancia hospitalaria inferior a los 
Gráfico 4. Distribución de los pacientes según número de regiones corporales afectadas. Unidad de Quemados del Hospital Luis Vernaza (Guayaquil, Ecuador). Período 2006 - 2010

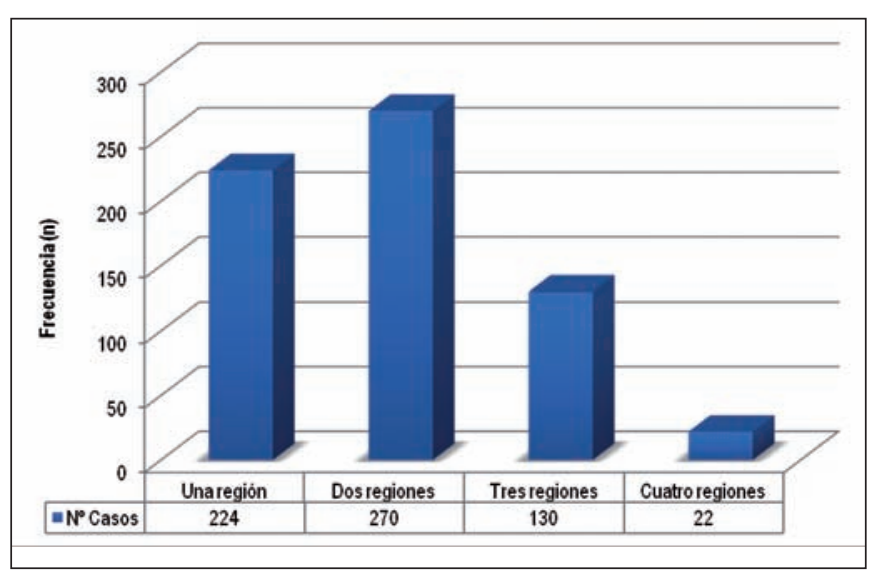

Gráfico 5. Clasificación de los pacientes según el tratamiento recibido. Unidad de Quemados del Hospital Luis Vernaza (Guayaquil, Ecuador). Período 2006 - 2010

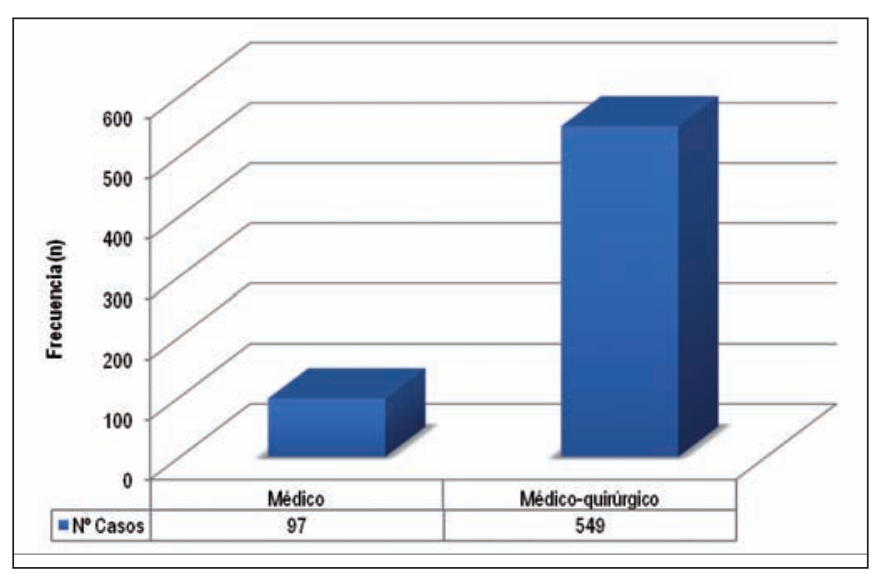

30 días y tan sólo el 1,6\% del grupo tuvo una estancia hospitalaria superior a los 45 días. El 15,3\% restante permaneció hospitalizado entre 30 y 44 días (Tabla I).

En el grupo de pacientes con quemaduras de grado moderado, la distribución de acuerdo al número de días de permanencia hospitalaria fue diferente, puesto que el $40,5 \%$ de los pacientes permaneció 30 días o más en el hospital, de los cuales tan solo 3 casos del grupo permanecieron ingresados más de 60 días. El 59,4\% restante tuvo una estancia inferior a los 30 días (Tabla I).

En los pacientes con quemaduras de grado mayor, la estancia media hospitalaria fue similar a la de los pacientes con quemaduras de grado moderado. El 63,5\% del grupo permaneció menos de 30 días en el hospital. En este grupo registramos 27 casos $(6,6 \%)$ que requirieron un ingreso igual o superior a 60 días, lo cual en términos absolutos y porcentuales, fue superior a la de la combinación de pacientes en esta misma situación catalogados como quemaduras de grado menor o moderado (Tabla I).

\section{Estancia hospitalaria según otros aspectos}

La edad media de los pacientes que permanecieron 60 días o más ingresados (38,03 $\pm 14,3$ años) no fue significativamente diferente a la de aquellos que permanecieron menos tiempo (36,06 $\pm 14,3$ años). La estancia media tampoco fue diferente considerando el género de los pacientes.

Aquellos pacientes con quemaduras con compromiso del $30 \%$ o más de la superficie corporal total tuvieron una estancia hospitalaria promedio significativamente mayor a la de aquellos pacientes con menor extensión de las lesiones ( 46,9 frente a 25,1 días; $\mathrm{p}<0,05)$.

Tabla I. Estancia hospitalaria según gravedad de los pacientes de acuerdo a los criterios de la American Burn Association. Unidad de Quemados del Hospital Luis Vernaza (Guayaquil, Ecuador). Período 2006 - 2010

\begin{tabular}{|c|c|c|c|}
\hline $\begin{array}{c}\text { ESTANCIA } \\
\text { HOSPITALARIA }\end{array}$ & $\begin{array}{c}\text { QUEMADURA } \\
\text { MENOR } \\
(n=124)\end{array}$ & $\begin{array}{c}\text { QUEMADURA } \\
\text { MODERADA } \\
(n=111)\end{array}$ & $\begin{array}{c}\text { QUEMADURA } \\
\text { MAYOR } \\
(n=411)\end{array}$ \\
\hline Edad media (años) & $37,3( \pm 16,1)$ & $36,39( \pm 14,4)$ & $35,7( \pm 13,5)$ \\
\hline Estancia media hosp. (días) & $19,3( \pm 10,4)$ & $28,9( \pm 14,47)$ & $28,3( \pm 23,7)$ \\
\hline$<15$ días [\%] & $44[35,5]$ & $15[13,5]$ & $90[21,9]$ \\
\hline 15-29 días [\%] & $59[47,6]$ & $51[45,9]$ & $171[41,6]$ \\
\hline 30-44 días [\%] & $19[15,3]$ & $26[23,4]$ & $87[21,2]$ \\
\hline 45-59 días $[\%]$ & $1[0,8]$ & $16[14,4]$ & $36[8,8]$ \\
\hline$\geq 60$ días $[\%]$ & $1[0,8]$ & $3[2,7]$ & $27[6,6]$ \\
\hline
\end{tabular}


Tabla II. Estancia hospitalaria de acuerdo a la presencia de quemaduras de tercer grado. Unidad de Quemados del Hospital Luis Vernaza (Guayaquil, Ecuador). Período 2006 - 2010

\begin{tabular}{|l|c|c|}
\hline \multicolumn{1}{|c|}{$\begin{array}{c}\text { ESTANCIA } \\
\text { HOSPITALARIA }\end{array}$} & $\begin{array}{c}\text { SIN } \\
\text { QUEMADUAS 3 } \\
(\mathbf{n = 3 7 1 )}\end{array}$ & $\begin{array}{c}\text { CON } \\
\text { QUEMADO } \\
(\mathbf{n = 2 7 5 )}\end{array}$ \\
\hline Estancia media hosp. (días) & $20,8( \pm 12,0)$ & $34,6( \pm 26,4)$ \\
\hline$<15$ días [\%] & $117[31,5]$ & $32[11,6]$ \\
\hline $15-29$ días [\%] & $180[48,5]$ & $101[36,7]$ \\
\hline $30-44$ días [\%] & $57[15,4]$ & $75[27,3]$ \\
\hline $45-59$ días [\%] & $11[3,0]$ & $42[15,3]$ \\
\hline$>60$ días [\%] & $6[1,6]$ & $25[9,1]$ \\
\hline
\end{tabular}

Gráfico 6. Distribución del número de muertes en la Unidad de Quemados según las categorías de quemaduras descritas por la American Burn Association. Unidad de Quemados del Hospital Luis Vernaza (Guayaquil, Ecuador). Período 2006 - 2010

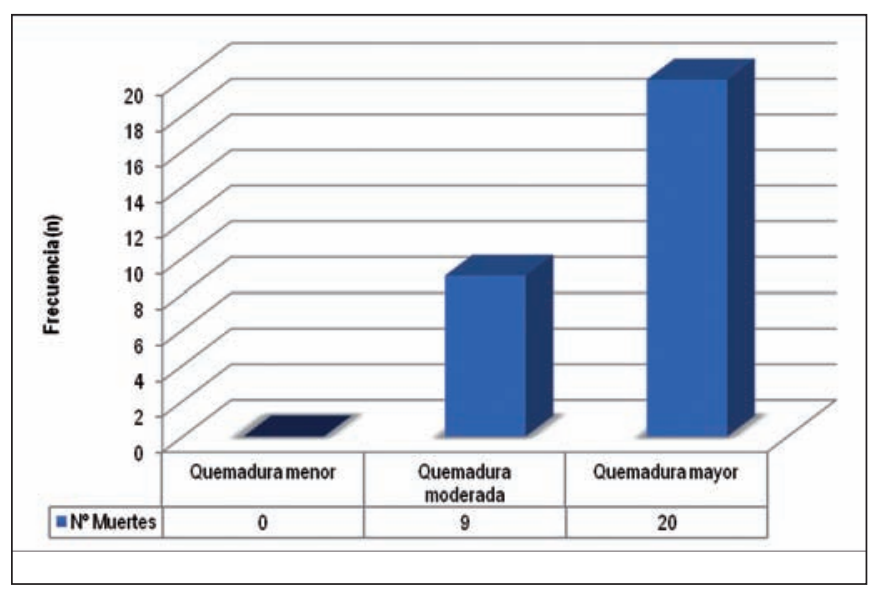

$\mathrm{Al}$ analizar la estancia media hospitalaria tomando en cuenta la presencia o no de quemaduras de tercer grado, sin importar su extensión, encontramos que la media en pacientes que sí tuvieron este tipo de lesiones fue de 34,6 días $( \pm 26,4)$, en comparación con tan solo 20,8 días $( \pm 12,0)$ en el grupo que no presentó dicha categoría de quemaduras (Tabla II).

\section{Mortalidad}

Registramos 29 fallecimientos (4,48\%). De ellos, el $68,9 \%(n=20)$ ocurrió en pacientes con quemadura mayor según los criterios ABA. El porcentaje restante sucedió en pacientes con categoría de quemadura moderada. No hubo fallecimientos entre los pacientes con quemaduras menores (Gráfico 6).

\section{Discusión}

En nuestro estudio encontramos un predominio de pacientes de sexo masculino, lo cual está respaldado por al- gunos estudios previos $(4,10-15)$, pero contrasta con la información obtenida por otros autores $(16,17)$. Al parecer, ciertos factores culturales y/o regionales, ejercerían algún efecto sobre el predominio por sexos de las lesiones por quemaduras $(13,14)$.

En nuestra revisión, la causa más común de quemaduras fue la lesión por llama directa, seguida por la debida a causas eléctricas y térmicas; estos hallazgos concuerdan con lo recogido en otros trabajos como los de Vargas Naranjo y col., y también González Alaña y col. $(4,11,12)$, y son diferentes a los recogidos en la literatura mundial, en los que la causa más frecuente es la térmica, seguida por la eléctrica, mientras que la llama directa aparece como el tercer agente causante más frecuente $(14,15,18)$. Esta discrepancia, probablemente sea atribuible al hecho de que las revisiones citadas abarcan todas las quemaduras atendidas en los centros hospitalarios, mientras que el presente trabajo se limita a analizar solamente a aquellos pacientes que requirieron tratamiento especializado en la Unidad de Quemados, excluyéndose los casos tratados ambulatoriamente. Epidemiológicamente, las quemaduras por llama y eléctricas son las más prevalentes en los países con bajos ingresos (4).

En nuestro estudio, el lugar más frecuente de los accidentes fue el domicilio, lo cual es respaldado por otros autores (15-17). Sin embargo, el lugar de trabajo también registró un número considerable de casos, concordando con los datos expuestos por Vargas Naranjo y col. (4). Probablemente esta discordancia se deba a las diferentes políticas y legislaciones de seguridad laboral en países más desarrollados que el nuestro. Por ello, es importante proponer revisar de manera más exhaustiva y global este escenario para poder generar propuestas orientadas a la prevención de estos accidentes en el ámbito laboral.

Las extremidades superiores, al igual que en el trabajo de Coban y col. (10), fueron la región corporal más 
frecuentemente afectada, lo cual puede explicarse debido al mecanismo de trauma mediante el cual suelen producirse las lesiones por llama y corriente eléctrica, causas más comunes en nuestro estudio. Estos datos discrepan con los recogidos por Maldonado y col., cuyos pacientes presentaron un mayor número de quemaduras en cara y cuello (11).

El abordaje médico de la mayor parte de los pacientes de nuestro grupo de estudio requirió algún tipo de cirugía debido a la extensión y/o a la profundidad de las lesiones que padecían. No hemos visto que la estancia hospitalaria se incrementara por el hecho de haber recibido algún tipo de cirugía; el tratamiento más frecuentemente realizado fue la limpieza quirúrgica con injerto de piel.

En cuanto a la relación entre la superficie corporal afectada y la estancia hospitalaria, encontramos que ésta última fue significativamente mayor en pacientes con afectación del $30 \%$ o más de su superficie corporal en comparación con aquellos pacientes con menor extensión de las lesiones. Cuanto más extensa es la superficie corporal total afectada, mayor es el riesgo de sufrir complicaciones, entre las cuales las infecciones intrahospitalarias juegan un papel muy importante; esto se debe a que en las quemaduras existen tejidos descubiertos y desvitalizados que hacen a los pacientes quemados más susceptibles a la colonización por bacterias (4), siendo muy frecuentes las infecciones intrahospitalarias entre este tipo de pacientes, con una tasa de afectación de $36,2 / 1000$ pacientes por día (4). Además, las quemaduras extensas causan un notable incremento de mediadores inflamatorios y catecolaminas, lo que conlleva a su vez un incremento de la tasa metabólica de estos pacientes (10).

Observamos un comportamiento similar al analizar la presencia de quemaduras de tercer grado. La estancia media en este grupo de pacientes fue muy superior en comparación a la de los pacientes que presentaron lesiones de menor grado. Además, encontramos una distribución de acuerdo a los días de estancia hospitalaria en la cual queda de manifiesto que el hecho de tener este tipo de lesiones puede incluso prolongar los días de hospitalización, tanto como una quemadura menos profunda, pero más extensa.

En los pacientes con quemaduras consideradas de grado moderado-mayor (según criterios $\mathrm{ABA}$ ) la estancia hospitalaria fue significativamente mayor que en el grupo de quemaduras de grado menor. Esto, como es de esperar, coincide con lo recogido por otros autores $(19,20)$. Sin embargo, no encontramos diferencias de consideración entre los grupos de pacientes con quemaduras de grado moderado y mayor; probablemente, esto se deba a que según la clasificación $\mathrm{ABA}$, todas las quemaduras eléctricas son consideradas como mayores, lo cual muchas veces no refleja la extensión y/o profundidad de las lesiones, datos que demostraron de manera independiente ser factores que predicen con cierta precisión la duración de la estancia hospitalaria.
La mortalidad en los pacientes quemados, sobre todo en los grandes quemados, sigue siendo en la actualidad uno de los retos más importantes a los que se enfrentan los profesionales de la salud. Dicha mortalidad se justifica durante la primera semana por el shock hipovolémico y la inhalación de humos que acompañan a las quemaduras y luego, por el shock séptico, las patologías directamente relacionadas con la extensión de las quemaduras y por la ausencia proporcional de zonas donantes para la cobertura de extensas superficies corporales expuestas (12). En esta revisión retrospectiva, sólo registramos 29 fallecimientos que ocurrieron durante las primeras horas de ingreso de los pacientes y se dieron en aquellos casos de extensa afectación de la superficie corporal. La mortalidad, morbilidad y discapacidad relacionadas con las quemaduras pueden prevenirse a gran escala a través de la educación de la población sobre medidas de seguridad, implementado códigos y legislaciones de seguridad laboral y creando unidades de atención especializadas para este tipo de lesiones.

Consideramos que la principal limitación del presente estudio fue su carácter retrospectivo, que condicionó el que las variables analizadas fueran solo las que estaban disponibles en los registros de la Unidad de Quemados. Resultaría de interés realizar un estudio similar que abarque otros aspectos, tales como la intencionalidad de la quemadura (accidente, suicidio, etc.) y con una mejor descripción de las circunstancias en las que ocurrió el incidente, para de esta manera, poder elaborar estrategias preventivas. También sugerimos la realización de un estudio destinado a valorar el grado de invalidez que resulta como secuela en estos catastróficos accidentes.

\section{Conclusiones}

Las lesiones por quemadura son culpables del trauma más serio y catastrófico que le puede ocurrir a un ser humano; estas injurias implican, en su mayoría, la necesidad de tratamiento por un equipo especializado de salud. El conocimiento de los agentes causantes de estas lesiones, los lugares donde más se producen, y las áreas corporales más comúnmente afectadas, nos proporcionan información útil para realizar programas preventivos y tomar acciones necesarias para evitar, en la medida de lo posible, la recurrencia de estas calamidades.

\section{Dirección del autor}

Dr. Rafael Guerrero Torbay

Urb. Río Grande Mz C30 Km 1,5 Via Samborondón CP. 091650

Guayaquil, Ecuador

e-mail: rafaelguerrerot@gmail.com 


\section{Bibliografía}

1. Castillo P. Quemaduras: Conceptos para el médico general. Cuad Cir (Valdivia). 2003;17(1):58-63.

2. Ramírez C, Ramírez C, Ramírez MA, Ramírez N. Manejo del paciente quemado. Salud UIS. 2007;39:124-136.

3. Pérez F, Vázquez E, Cepero R, Pina E, Ramírez E. Características epidemiológicas de las quemaduras asistidas en la Unidad de Quemados del Hospital Provincial de Ciego de Ávila (Internet). MediCiego; 2005 (citado Mayo 8, 2010). Disponible en: http://www.bvs.sld.cu/revistas/mciego/vol11_02_05/articulos/a19_v11_0205.html

4. Vargas Naranjo, S. et al. Factores de riesgo para el desarrollo de infecciones intrahospitalarias en pacientes quemados. Hospital S. Juan de Dios, Costa Rica: Enero 2003-Diciembre 2005. Parte I. Cir plást iberolatinoam 2009;35(3): 223-232.

5. Shukla PC, Sheridan RL. Initial Evaluation and Management of the Burn Patient (Internet). eMedicine; 2008 (citado Mayo 8, 2010). Disponible en: http://emedicine.medscape.com/article/435402-overview

6. Gallagher JJ, Wolf SE, Herndon DN. Burns. En: Townsend CM, editor. Sabiston Textbook of Surgery: the biological basis of modern surgical practice. $18^{\circ}$ ed. Philadelphia, PA: Saunders Elsevier; 2008. Pp 559-585.

7. Holmes JH, Heimbach DM. Burns. En: Brunicardi FC, Andersen DK, Billiar TR, et. al, editores. Schwartz's Principles of Surgery. 9th ed. New York, NY: McGrawHill; 2009. Pp 189-223.

8. American Burn Association. Hospital and prehospital resources for optimal care of patients with burn injury: guidelines for development and operation of burn centers. J Burn Care Rehabil 1990;11:98-104.

9. Pérez M, Lara J, Ibañez J. Guía de actuación ante el paciente quemado (Internet). Hospital R.U. Carlos Haya Málaga - Servicio Andaluz de Salud. (Citado 22 de Abril de 2011). Disponible en: http://www.carloshaya.net/denfermeria/media/gpq.pdf

10. Coban YK, Erkiliç A, Analay H. Our 18-month experience at a new burn center in Gaziantep, Turkey. Ulus Travma Acil Cerrahi Derg 2010;16(4):353-356.
11. Maldonado AA, Küntscher M, Sillero A. Análisis de parámetros bioquímicos en grandes quemados: nuevos factores pronósticos. De la investigación básica a la clínica. Cir plást iberolatinoam 2012;38(4):305-312.

12. González Alaña I, Aguilar Barrón P, Torrero López JV, Ferreiro González I, Gabilondo Zubizarreta FJ. Cobertura de grandes quemados con cultivo de queratinocitos: casuística de nuestra Unidad y protocolo de tratamiento. Cir plást iberolatinoam 2012:38(3):257-264.

13. Anlatici R, Ozerdem OR, Dalay C, Kesikta E, Acartürk S, Seydao lu G. A retrospective analysis of 1083 Turkish patients with serious burns. Burns 2002;28:231237.

14. Lari AR, Alaghehbandan R, Nikui R. Epidemiological study of 3341 burns patients during three years in Tehran, Iran. Burns 2000;26:49-53.

15. Tang K, Jian L, Qin Z, Zhenjiang L, Gomez M, Beveridge M. Characteristics of burn patients at a major burn center in Shanghai. Burns 2006;32:1037-1043.

16. Fernández-Morales E, Gálvez-Alcaraz L, FernándezCrehuet et al. Epidemiology of burns in Malaga, Spain. Burns 1997;23:323-332.

17. Hemeda M, Maher A, Mabrouk A. Epidemiology of burns admitted to Ain Shams University Burns Unit, Cairo, Egypt. Burns 2003;29:353-358.

18. Tung KY, Chen ML, Wang HJ, Chen GS, Peck M, Yang J, Liu CC. A seven-year epidemiology study of 12,381 admitted burn patients in Taiwan-using the Internet registration system of the Childhood Burn Foundation. Burns 2005;31:S12-17.

19. Pavoni V, Gianesello L, Paparella $L$ et al. Outcome predictors and quality of life of severe burn patients admitted to intensive care unit. Scand J Trauma Resusc Emerg Med. 2010, 27;18:24-31.

20. Vendrusculo TM, Balieiro CR, Echevarría-Guanilo ME et al. Burns in the domestic environment: characteristics and circumstances of accidents. Rev Lat Am Enfermagem. 2010;18(3):444-451. 